\title{
Mesenhymal stem cells in treatment of pyoderma gangrenosum in patient with ulcerative colitis: clinical case
}

\begin{abstract}
Inflammatory bowel diseases: ulcerative colitic (UC) and Croh's disease are serious medical problem in connection with plenty of complications and extraintestinal manifestations (EIM). Presents of EIM, especially reactive skin lesions which correlated with high activity of UC is predicted to severe clinical course, hospitalizations, immunomodulators and steroids prescription. Immunosupressive therapy sometimes is not effective and we need to have new methods to escalate immunomodulation in these patients. One of this method is mesenhymal stem cells transplantation (MSCT). At the same time experience and efficacy of MSCT in UC cohort is controversial here we present a clinical case of acute severe ulcerative colitis with EIM pyoderma gangrenosum to represent an interesting clinical peculiarities and response to the therapy.

Patient $\mathrm{S}$ is a 36-year-old, male complained of bloody diarrhea and moderate abdominal pain. He describes up to 4-7 urgent stool per day and 3-5 stool per night weight loss. During last 2 week patient had seen local skin redness in left arm, at last 5 days in this area patient sow ulceration and feel pain. Stool culture was negative for infection. Colonoscopy and biopsies was performed, which show erythemae, friability of colon mucosa with erosions and ulcers. Histologicaly diagnosis of ulcerative colitis was confirmed. Dermatologist diagnosed pyoderma gangrenosum multifocal localization ulcerative subtypes.

Treatment for presumed ulcerative colitis is initiated with mesalazine $7 \mathrm{~g}$ daily: $3 \mathrm{~g}$ orally, $4 \mathrm{~g}$ per rectum and prednisone at $60 \mathrm{mg}$ orally daily. After 3 days treatment patient's conditions did not become better. Intravenous steroids have been prescribed - 16mg of dexamethasone. Infliximab $5 \mathrm{mg} / \mathrm{kg}$ were administered, one infusion was performed and azathioprine $150 \mathrm{mg}$ per day was prescribed. But after 28 days of treatment patient had 5-6 times stool per day and 1-2 per night 1-3 times a day with blood. Skin lesions didn't change significantly. Autologous mesenhymal stem cells transplantation was performed. After 12 months patient obtained monotherapy of mesalasine $2.0 \mathrm{~g}$ per day and still has stable remission. During last 2 years patient didn't obtained any treatment and don't have exacerbations of UC.
\end{abstract}

Keywords: severe ulcerative colitis, pyoderma gangrenosum, mesenhymal stem cells transplantation clinical case

\section{Background}

The inflammatory bowel diseases (IBD) are chronic relapsing diseases of the gut. Ulcerative colitis (UC) belongs to a group of IBD with autoimmune character that cause inflammation in the colon with common symptoms such as bloody diarrhea and abdominal pain. ${ }^{1,2}$ In UC patients part of colon or all mucosa in large bowel are affected. Usually patients with UC has an intermittent course of disease with periods of exacerbation and remission. At the same time, development of acute, severe UC contributes more careful examination because more often turns to complications and more widely presented by extraintestinal manifestations (EIM). ${ }^{3}$ Skin lesion correlated with activity and severity of UC. Cutaneous manifestation in IBD classified into three groups: granulomatous EIM which have the same histological features as a bowel disease (perianal fistulas, epidermolisis bullosa acquisita) and more typical for Chroh's disease, reactive EIM (erythema nodosum and pyoderma gangrenosum) which are more common in UC patients and secondary skin EIM related to nutrition deficiency. ${ }^{4}$ Pyoderma gangrenosum (PG) is an ulcerative lesion which correlated with severity and exacerbations of UC, but manifested longer, after the exacerbation of the bowel
Volume 10 Issue I - 2019

\author{
AE Dorofeyev,' AG Popandopulo, ${ }^{2}$ AA \\ Dorofeyeva, ${ }^{3}$ Yu Z Dynia,' PP Snisarevsky' \\ 'National Medical Academy of Postgraduate Education \\ n.a.P.L.Shupik, Ukraine \\ 'International Center of Biotechnology "BIOSTEM", Ukraine \\ ${ }^{3}$ Gerontology Institute n.a.D.F.Chebotarev National Medical \\ Academy, Ukraine
}

Correspondence: AE Dorofeyev, National Medical Academy of Postgraduate Education n.a.P.L.Shupik, Kiev, Ukraine, Email dorofeyevand@gmail.com

Received: February 22, 2018 | Published: February 28, 2019 disease. PD reported incidence in 1-4\% of UC patients, but treatment is difficult with high doses of systemic steroids, immunomodulators and immunobiologics. ${ }^{3,4}$ Mesenhymal stem cells (MSC) therapy is one of the methods on immunosuppressive treatment in autoimmune diseases, but efficacy of MSC transplantation in UC and especially in PD still controversial. ${ }^{5}$ As far as this question remains still unclear here we present a clinical case of of acute severe ulcerative colitis with pyoderma gangrenosum to represent an interesting clinical peculiarities and response to the MSC therapy.

\section{Clinical case}

Patient S. is a 36-year-old, male, high technical education, an engineer was examined by gastroenterologist and directed to our university clinic with 23 days of bloody diarrhea and moderate lower abdominal pain. He complains of liquid, mixed with blood urgent stool up to 4-7 times per day and 3-5 stools per night. Patient described weight loss near $5 \mathrm{~kg}$ during last 4 week. At last 12 moths he had 3 diarrhea episodes, but without blood, that's why he didn't visited doctor previously. The detailed medical anamnesis was taken. Patient said that this episode of disease started suddenly with bloody diarrhea 
and night defecation. During last 2 week patient had seen local skin redness in left arm, at last 5 days in this area patient sow ulceration and feel pain. He felt subfebrile temperature during last week. Patient did not obtained antibiotic or non-steroidal anti-inflammatory drug. Patient is a lifelong nonsmoker and has no history of medical illness or surgery.

The patient was hospitalized in gastroenterological department for detailed examination. Diagnosis of UC was based on clinical symptoms, endoscopic picture and histological investigation. During physical examination patient has suffered from abdominal pain syndrome with moderate intensity. Also patient described episodes of increasing pain intensity after defecation. Average duration of pain syndrome was $9.1 \pm 2.2$ hours per day. In left arm of patient skin has painful multiple lesions one with erythematous pustules, other with burrowing ulcer with violaceous edges and undermined borders (Figure 1). Other organs and systems were in normal condition. Stool culture was negative for infection, but fecal leukocytes were positive in a moderate quantity.

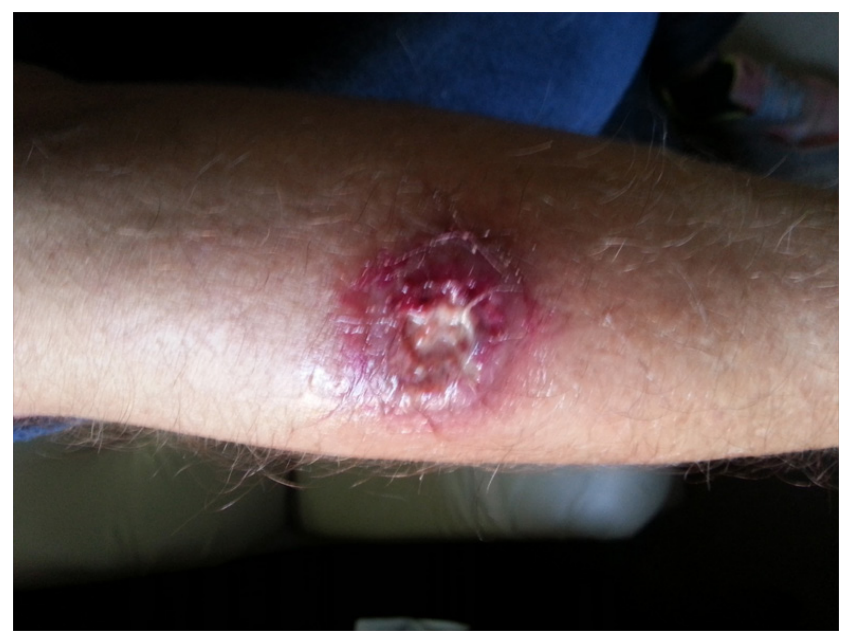

Figure I Patient S, burrowing ulcer with violaceous edges and undermined borders in left arm.

Severity of UC correlated with extensive inflammation in colon. Activity of UC according to Rachmilewitz activity index (CAI). At our patient CAI was 9.0 score points. Colonoscopy and biopsies was performed, which show erythemae, friability of colon mucosa with erosions and ulcers. Endoscopically diagnosis of ulcerative pancolitis with high activity was performed (Figure 2). Bioptates of colon mucosa were stained by hematoxylin-eosin, alcian blue at $\mathrm{pH} 1.0$ and 2.5 for determination of sulfated and non-sulfated glucosaminoglycans and glycoproteins and for goblet cells and colon mucus. To characterize the mucus production, the PAS-reaction was performed. During histological evaluation severe inflammation with high cell infiltration in colon mucosa, crypt-abscesses (Figure 3); reducing quantity of goblet cells were found. Reduction of goblet cells was revealed from moderate to total depletion of goblet cells with small vacuoles and low content of mucus. Moreover, significant qualitative mucus modification has been identified. The PAS-reaction showed a low level of PAS-positive substances. That indicates a significant depletion of mucus production and abnormal glycosylation which decrease the protective function of mucus layer in UC. Active leucocytes containing glycogen were found. Alcian blue at $\mathrm{pH} 1.0$ revealed weak staining. However, at $\mathrm{pH} 2.5$ a moderate intensive staining was found. These findings indicate imbalance between sulfated and unsulfated glucosaminoglycans that results in the decreasing of protective function of the mucus barrier typical for UC.

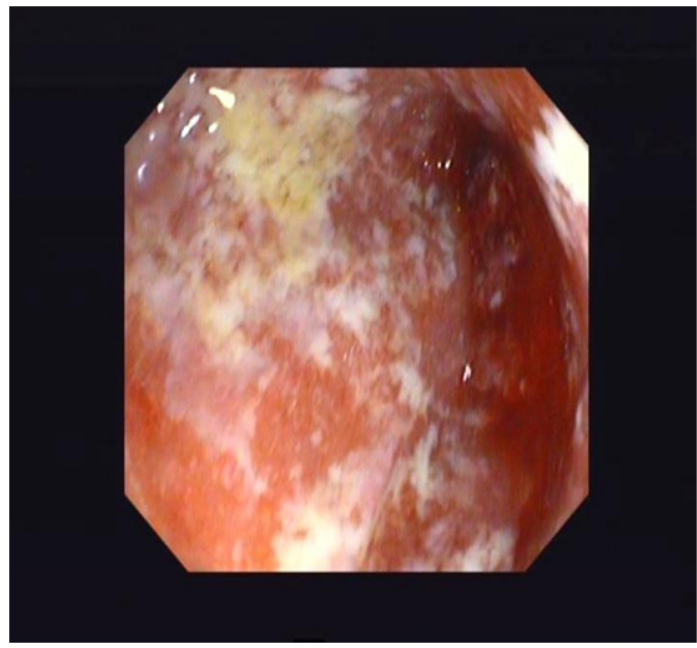

Figure 2 Flexible colonoscopy in patient $\mathrm{S}$, friable and erythematous mucosa with erosions and ulcers.

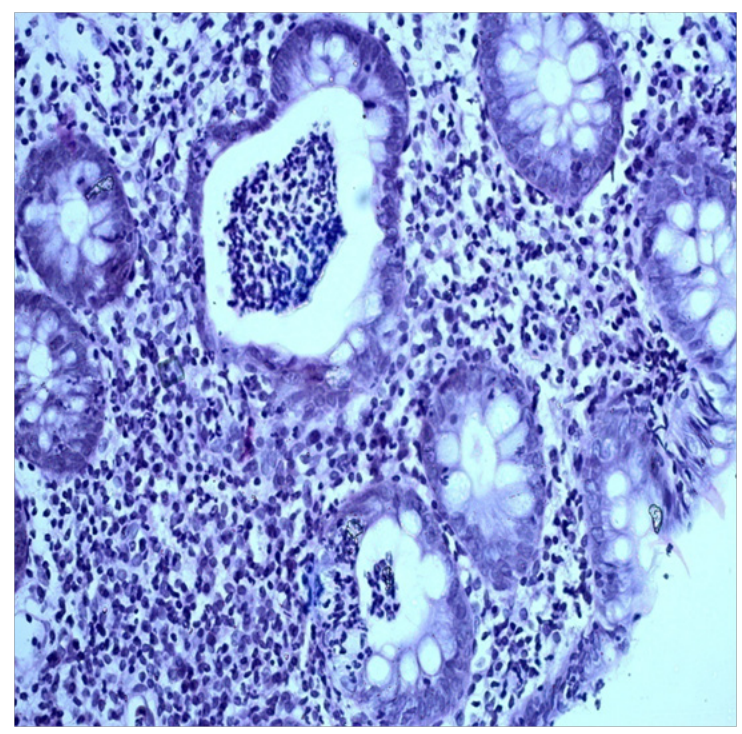

Figure 3 Infiltration in colon mucosa, crypt-abscess in patient S., hematoxylineosin staining $(200 x)$.

Consultation of dermatologist was performed. During this consultation skin biopsy from ulcer lesion was done. Histological result describe entotelial swelling and injury with fibrinoid necrosis, perivascular lymphocytic infiltrate with presents of active neutrophils. Due to clinical picture and morphological result diagnosis of pyoderma gangrenosum multifocal localization ulcerative subtypes was done.

According to clinical, endoscopical and histological results, dermatologist consultation patient $\mathrm{S}$ has diagnosis: Ulcerative colitis, pancolitis (E3), active, severe form (S3) with extraintestinal manifestation (pyoderma gangrenosum). Treatment for presumed ulcerative colitis is initiated with mesalazine $7 \mathrm{~g}$ daily: $3 \mathrm{~g}$ orally, $4 \mathrm{~g}$ per rectum and prednisone at $60 \mathrm{mg}$ orally daily. Patient's condition changed not significantly. Still he had moderate abdominal pain syndrome and diarrhea. After 72 hours stool frequency was 8 times per day (3 per night) with urgency, blood in stool occasionally. Intravenous steroids have been prescribed in dose $16 \mathrm{mg}$ of dexamethasone. 
Five days later patient felt better. Stool frequency became 7 times per day, 1-2 per night with trace blood in stool. General well being has increased. Patient gained $1 \mathrm{~kg}$. Mood and night sleep improved, but on the skin of the back two new focus of PG were found. At the same time, major progress in treatment was not achieved. After 14 days patient's condition did not change significantly. Infliximab $5 \mathrm{mg} / \mathrm{kg}$ was administered and after first infusion stool frequency decreased. Patient had 6 times stool per day, sometimes with blood in stool 2-3 time per day. Also he didn't mention urgency and night diarrhea. Azathioprine $150 \mathrm{mg}$ per day was prescribed. But after 28 days of treatment patient had 5-6 times stool per day and 1-2 per night 1-3 times a day with blood. Skin lesions didn't change significantly. Patient has been consulted by coloproctologist and decision of operation - colectomy was discussed. A consilium was held with the participation of a gastroenterologist, a coloproctologist, an endoscopist, an immunologist, and a decision was made to conduct MSC transplantation.

\section{MSC preparation and transplantation method}

Mesenchymal stem cells $(0.5-1 \mathrm{~mL})$ obtained by puncture of breast bone of patient under local anaesthesia in strictly sterile conditions, which were kept during further work with the cells in the culture box. After deposition on red blood cells during 1-2 hours at room temperature, supernatant fluid was aspirated from bone marrow cell suspensions by Pasteur pipette, received cells were put into substance 199. Obtained suspension of cells were centrifuged during 10 minutes under 1000rpm till precipitate was received, which was resuspended in growth environment. RPMI-1640 medium which contains penicillin $(100 \mathrm{IU} / \mathrm{ml})$, amphotericin $(100 \mathrm{ng} / \mathrm{ml})$, L-Glutamine $(2 \mathrm{mM})$ and $20 \%$ of fetal bovine serum were used.

Cultivation was carried out in Carrel's T-flask with a bottom area of $25 \mathrm{~cm}^{2}$, they put $5 \times 10^{6}-10^{7}$ bone marrow cell in $8 \mathrm{ml}$ of growth medium. The T-flasks were flushed with a gas mixture, that contains $5 \%$ of carbon dioxide and $95 \%$ of air, and placed in a thermostat at $37{ }^{\circ} \mathrm{C}$. This procedure was made each time once a medium was changed or cells were transferred into new T-flask. When the confluent monolayer was reached, the cells were resuspended using $0.25 \%$ trypsin solution in new vials, first with the same bottom area $\left(25 \mathrm{~cm}^{2}\right)$, and subsequently - during cell mass increasing - with a 175 $\mathrm{cm}^{2}$ bottom area. This cultivation method made it possible to obtain a mesenchymal stem cell population in the amount of $(1.5-2) \times 10^{8}$ cells necessary for transplantation of the original bone marrow by the end of the $5^{\text {th }}$ week.

The culture of MSC was injected intravenously once. To perform systemic transplantation of 150-200 million MSCs propagated in culture, cells were weighed into $200 \mathrm{ml}$ of sterile saline containing heparin at a concentration of $50 \mathrm{U} / \mathrm{ml}$, and administered by a dropper to the patient for 40-60 minutes. Before MSC injection, the dose of previously assigned aminosalicylates was kept stable during 4 weeks. Patient obtainer mesalasine at $6.0 \mathrm{~g} /$ day, the dose of glucocorticosteroids was reduced by half, patient received prednisone $30 \mathrm{mg} /$ day, and Asatioprin before 10 days the transplantation was canceled, infliximab was stopped 4 weeks before MSC transplantation.

After the MSC transplantation the patient felt better, in three weeks patient well being had improved: stool frequency reduced till 1-2 per day without blood and urgency, he had no night defecation, body weight became normal. Patient had no complains about skin signs. Focuses PG in a back completely epithelized without cicatricial changese, PG ulcer in arm improved, but complete reparation was observed in week 5 after transplantation only. After 12 months patient obtained monotherapy of mesalasine $2.0 \mathrm{~g}$ per day and still has stable remission. During last 2 years patient didn't obtained any treatment and don't have exacerbations of UC. 39 month after MSC transplantation patient visited gastroenterologist with complaints for stool 2-4 times a day without blood, skin was completely normal. Flexible colonoscopy and biopsies was performed, which show not significant changes in colon mucosa, normal vascular pattern without any erosions and ulcers. Morphologically ulcerative colitis in remission were observed. Mesalasine $2.0 \mathrm{~g}$ per day were recommended and after 4 weeks stool 1 time a day, without any other complaints.

\section{Discussion}

In ulcerative colitis skin is a site which frequent involved with extraintestinal manifestations. Reported frequencies of skin EIM in UC patient are variable, but ranging from $6 \%$ to $43 \% .{ }^{6,7}$ Presents skin EIM correlated with severity of UC and significantly decreased quality of life in UC patients. Treatment of patient with UC and extraintestinal manifestation is often challenged by concomitant medication use and comorbid conditions that may affect on the induction and maintenance therapies. In UC patients with reactive skin EIM, pyoderma gangrenosum is necessary to escalate immunosupression and increase dose of systemic steroids, immunomodulators or immunobiologics. In these patients are at increased risk for steroid-related complications, and in combination with immunosupressive therapy it may lead to unfavorable side effects. ${ }^{8,9}$ The clinical course and therapeutic response in UC patients with PG are not well defined. Some studies reported that these patients were more likely to present a limited extent but more severe course at the onset of disease. Moreover, some results also suggested that immunobiologics (infliximab) was associated with improvement of UC, but not in PG. That's why new immunosuppressive strategy in patients with $\mathrm{UC}$ and PG is very perspective. Mesenhymal stem cells have immunosuppressive and immunomodulative activity. These cells prevent the T-cell response in not by direct means by modulating the dendritic cells and disturbing the function of NK-cells and $\mathrm{CD}_{8+}, \mathrm{CD}_{4+}$ response. MSC produce leukemia inhibitory factor (LIF) is the most pleiotropic member of the interleukin- 6 family of cytokines which modified synthesis of proinflammatory interleukins IL- $6,-7,-11,-12,-14,-15,-27.5,10,11$ MSC migrate in inflammation area due to chemotaxis gradient and induce a local suppressive microenvironment by production colony stimulating factor 1 (macrophage-specific) and stem cells factor. Promising results demonstrate the functional ability of the potential of MSCs to influence the immune response and dampen inflammation, affirming their clinical practicality for therapeutic transplantation in autoimmune diseases. ${ }^{12,13}$ There has been inconsistent yet promising results from clinical trials in the past few decades, using stem-cell therapy through hematopoietic stem cells or mesenchymal stem cells. Autologous stem-cell therapy can have less side-effects than allogeneic MSC treatment. Transplant data from 2013 show that over 320,000 patients have received either autologous or allogeneic stem-cell transplants for various indications. ${ }^{14,15}$ There are only a few studies reporting mesenchymal stem cell transplantation (MSCT) in IBD patients, especially in UC and several publications about MSCT in patients with pyoderma gangrenosum, but not in combination ogf these diseases. ${ }^{16-18}$

In this clinical case we tried to analyze some peculiarities of clinical course in patient with $\mathrm{UC}$ and PG the consequences of standard 
treatment algorithm and MSCT applied for this patient. Patient $\mathrm{S}$ had severe course of UC, which was confirmed by histological examination. Massive leukocytes infiltration, goblet cells depletion and low mucine production indicate significant decreasing of mucosal barrier and reducing its protective function in severe UC. More over patient suffered from extraintestinal manifestation multifocal ulcerative subtype of pyoderma gangrenosum, which significantly decreased quality of life and was potential focus for microbial superinfection complications. Pancolitis and severe changes of large bowel mucosal barrier increase immune changes and dysfunction. Immune system become more sensitive to foreign agents, infections of mucosal surfaces become more common, side-effects and complication frequent. In this situation treatment strategy could be aggressive with high dose of systemic steroids, immunomodulators, biologics.

\section{Conclusion}

In our clinical case patient had received infliximab, azathioprine and oral and intravenous steroids. ${ }^{6-10}$ Although the treatment strategy corresponded to the contemporary rules of management patients with UC, we supposed that immunosupressive effect was lower than we expected. Patient has not significant clinical effect after 10 days of intravenous steroid application that's why we consider that patient has steroid refractory disease, which is a relative indication for surgical operation. ${ }^{19,20}$ Unfortunately, azatioprin and infliximab in this patient was not effective and we have only two options MSCT or colectomy. Probably, an effective response to MSCT and good outcome of UC caused by severe immune response during transplantation and short duration of the disease in this patient. At the same time, despite of acute severe colitis with extraintestinal manifestation- pyoderma gangrenosum, patient showed excellent outcomes and improved deep, steroid-free remission of UC at 39 month after diagnosis. In patient $\mathrm{S}$ mesenhymal stem cells transplantation modified natural history of the ulcerative colitis. This case report pushes to the deep investigation of the opportunity of mesenhymal stem cells transplantation in autoimmune disease patients, especially in UC with or without pyoderma gangrenosum and its management. Further research of pathogenic mechanisms of MSCT in UC and PG will improve our understanding of management of such patients.

\section{Acknowledgments}

None.

\section{Conflicts of interest}

Author declares no conflicts of interest.

\section{References}

1. Silverberg MS, Satsangi J, Ahmad T, et al. Toward an integrated clinical, molecular and serological classification of inflammatory bowel disease: report of a Working Party of the 2005 Montreal World Congress of Gastroenterology. Can J Gastroenterol. 2005;19:5A-36A.

2. Faubion WA, Loftus EV, Harmsen WS, et al. The natural history of corticosteroid therapy for inflammatory bowel disease: a populationbased study. Gastroenterology. 2001;121(2):255-260.
3. Piront $\mathrm{P}$, Louis E, Latour $\mathrm{P}$, et al. Epidemiology of inflammatory bowel diseases in the elderly in the province of Liege. Gastroenterol Clin Biol. 2001;26(2):157-161.

4. Rothfuss KS, Stange EF, Herrlinger KR. Extraintestinal manifestations and complications in inflammatory bowel disease. World J Gastroenterol. 2006;12(30):4819-4831.

5. Prodinger CM, Reichelt J, Bauer JW, et al. Current and Future Perspectives of Stem Cell Therapy in Dermatology. Ann Dermatol. 2017;29(6):667-687.

6. Ng SC, Kamm MA. Therapeutic strategies for the management of ulcerative colitis. Inflamm Bowel Dis. 2009;15(6):935-950.

7. Cerveny P, Bortlik M, Kubena A, et al. Nonadherence in inflammatory bowel disease: results of factor analysis. Inflamm Bowel Dis. 2007;13(10):1244-1249.

8. Higgins PD, Rubin DT, Kaulback K, et al. Systematic review: impact of non-adherence to 5-aminosalicylic acid products on the frequency and cost of ulcerative colitis flares. Aliment Pharmacol Ther. 2009;29(3):247-257.

9. Gisbert JP, Nino P, Cara C, et al. Comparative effectiveness of azathioprine in Crohn's disease and ulcerative colitis: prospective, longterm, follow-up study of 394 patients. Aliment Pharmacol Ther. 2008;28(2):228-238.

10. Rutgeerts P, Sandborn WJ, Feagan BG, et al. Infliximab for induction and maintenance therapy for ulcerative colitis. $N$ Engl $J$ Med. 2005;353(23):2462-2476.

11. Nicola NA, BabonJJ. Leukemia inhibitory factor (LIF). Cytokine Growth Factor Rev. 2015;26(5):533-544.

12. Murphy JM, Young IG. IL-3, IL-5, and GM-CSF signaling: crystal structure of the human beta-common receptor. Vitam Horm. 2006;74:130 .

13. Lennartsson J, Rönnstrand L. Stem cell factor receptor/c-Kit: from basic science to clinical implications. Physiol Rev. 2012;92(4):1619-1649.

14. Duran NE, Hommes DW. Stem cell-based therapies in inflammatory bowel disease: promises and pitfalls. Therap Adv Gastroenterol. 2016;9(4):533-547.

15. Ankrum J, Ong J, Karp J. Mesenchymal stem cells: immune evasive, not immune privileged. Nat Biotech. 2014;32:252-260.

16. Lazebnik L, Knyazev O, Parfenov A, et al. Optimization of cell therapy in patients with inflammatory bowel diseases. Ter Arkh. 2012;84(8):1017.

17. Lazebnik LB, Lychkova AE, Knyazev OV. Treatment of experimental ulcerative colitis. Bull Exp Biol Med. 2012;153(6):889-892.

18. Bittencourt MCB, Atanazio MJ, Xavier EM, et al. Postsurgical pyoderma gangrenosum after an autologous stem cell transplantation for multiple myeloma. BMJ Case Rep. 2018;2018.

19. Porro GB. Alimentary pharmacology \& therapeutics. 2007;26(6):779794.

20. Winther KV, Jess T, Langholz E, et al. Survival and cause-specific mortality in ulcerative colitis: follow-up of a population-based cohort in Copenhagen County. Gastroenterology. 2003;125(6):1576-1582. 